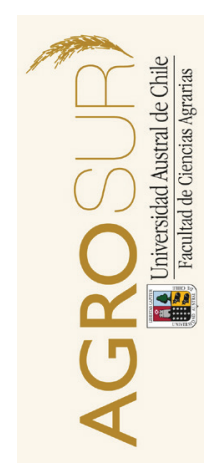

A RT ICLE INFO

Article history:

Received 09.02.15

Accepted 06.05.15

Keywords:

Wool quality

NIRS

Original Research Article,

Animal Science

*Corresponding author:

Daniel Alomar

E-mail address:

dalomar@uach.cl

\title{
Predicción de la calidad de lana mediante Espectroscopía de Reflectancia en el Infrarrojo Cercano (NIRS)
}

\author{
Prediction of wool quality by Near Infrared \\ Reflectance Spectroscopy (NIRS) \\ Alomar, D. ${ }^{a}$, Alarcón, M. $^{a}$, Kusanovic, A. ${ }^{b}$ \\ ${ }^{a}$ Instituto de Producción Animal, Universidad Austral de Chile. Casilla 567, Valdivia, Chile. \\ ${ }^{b}$ Escuela de Graduados, Facultad de Ciencias Agrarias, UACh.
}

\begin{abstract}
A B S T R A C T
This study was performed to evaluate the potential of Near Infrared Spectroscopy (NIRS) to predict some economically relevant variables of sheep wool, such as average fiber diameter (DMF), traction resistance (RT), scouring percentage (RL), comfort factor (FC) and staple length (LM). Corriedale and Multi Purpose Merino (MPM) wool samples (22 of each genotype) were scanned with a NIRSystems 6500 monochromator. To obtain the reference data, samples were analyzed with an OFDA 2000 instrument (Consorcio Ovino S.A.) and using the Sirolan Laser scan technique (New Zealand Wool Testing Authority, NZWTA Laboratory). Regression equations (WINISI II software) were obtained for the spectral data and the different variables of wool quality, by using modified partial least squares (MPLS) applying different mathematical treatments to the spectral values. The best equations were selected based on the determination coefficient of cross validation $\left(\mathrm{R}^{2} \mathrm{CV}\right)$, the standard error of cross validation (SECV) and the relation RPD, i.e., between standard deviation of reference data and the SECV. Three out of the five wool variables (DMF, RL and FC) could be reliably predicted by the NIR spectra, with calibrations that achieved a RPD $>3$ (DMF and FC) or very close to 3 (RL). No reliable equations could be obtained for RT and LM. It is concluded that NIRS has the capability to predict some wool variables of economic relevance, such as fiber diameter, comfort factor and scouring percentage.
\end{abstract}

\section{RESUMEN}

El presente estudio evaluó la posibilidad de predecir algunas variables lanimétricas relevantes, tales como diámetro medio de fibra (DMF), resistencia a la tracción (RT), rendimiento al lavado (RL), factor de confort (FC) y largo de mecha (LM), mediante espectroscopía de reflectancia en el infrarrojo cercano (NIRS). Se obtuvieron los espectros NIRS de muestras de lana de ovejas Corriedale y Merino Multipropósito (MPM), 22 de cada genotipo, mediante un monocromador NIRSystems 6500. Los datos de referencia se obtuvieron con un equipo OFDA 2000 (Consorcio Ovino S.A.) y mediante la técnica Sirolan Laserscan (New Zealand Wool Testing Authority, NZWTA). Se desarrollaron regresiones (cuadrados mínimos parciales modificados, MPLS) usando el software WINISI II, entre los espectros y las variables lanimétricas. Las ecuaciones se seleccionaron según su coeficiente de determinación de la validación cruzada $\left(\mathrm{R}^{2} \mathrm{CV}\right)$, el error estándar de validación cruzada (SECV) y la relación RPD, es decir, la relación entre la desviación estándar de los datos de referencia y el SECV.

Tres de las variables (DMF, RL y FC) pudieron ser predichas en forma confiable, con una relación RPD >3 (DMF y FC) o muy cercana a 3 (RL). Para las variables lanimétricas LM y RT no fue posible obtener ecuaciones de predicción confiables. Se concluye que NIRS tiene la capacidad de predecir algunas variables relevantes de calidad de lana, tales como el diámetro de las fibras, el factor de confort y el rendimiento al lavado.

Palabras clave: calidad de lana, NIRS.

\section{INTRODUCCIÓN}

La lana es una fibra natural muy valorada por la industria textil, que posee cualidades como ser renovable, sustentable, biodegradable, de bajo impacto ambiental y eficiente en el uso de energía. Es un retardante del fuego, protege de los rayos ultravioletas, reduce la electricidad estática y otorga resistencia térmica. Además tiene características saludables, ya que es una fibra "respirable", controla la humedad, es poco alergénica, absorbe tóxicos químicos y reduce el sonido (International Wool Textile Organisation, IWTO, 2012). Existe una gran diversidad de razas ovinas que producen diferentes clases y calidades de lana, obtenidas luego de siglos de selección (The Livestock Conservancy, 2015). Por su origen biológico, existe una considerable variación en las características de las fibras, tanto entre como dentro de los vellones (Itenge et al., 2009). 
En la Región de Magallanes y Antártica Chilena, tradicionalmente ha predominado la raza Corriedale (Fundación Chile, 2002). Esta raza fue formada por medio de cruzamientos en la isla sur de Nueva Zelandia, con el propósito de tener un ovino más robusto que el Merino, con un vellón amplio, pesado, de mechas más largas y con un equilibrio entre la producción de carne y lana (Asociación Argentina Criadores de Corriedale, 2007).

El Merino Multipropósito (MPM) es un biotipo de Merino originado en Australia con el objetivo de obtener una producción de lana más fina, de mayor calidad y a su vez, aumentar la producción de carne. El vellón MPM tiende a destacar además, por su alta densidad y longitud de fibras, en relación al Merino promedio. La calidad de la lana está determinada por diferentes propiedades de la fibra que son importantes en la industria textil. La principal es el diámetro de las fibras, pues las lanas más finas se destinan a prendas de vestir de alto valor. También son importantes la variabilidad del diámetro, el largo de la mecha y la resistencia a la tracción, es decir, que no presente estrangulaciones o debilidades en su crecimiento (Latorraca, 2005; Buxade, 1996). El Diámetro Medio de la Fibra (DMF) es el principal determinante del precio de la lana, debido a su influencia en la capacidad de producción de hilados, su naturaleza, tacto y toque de las prendas producidas con ellas. El diámetro de fibra de la lana puede ser medido usando diferentes tecnologías, tales como el microscopio de proyección (método de referencia) y otros de tipo secundario, tales como Airflow, OFDA y Sirolan Laserscan; que presentan la ventaja de ser más rápidos, precisos y con menor variabilidad entre operarios que el de referencia (Sacchero, 2005; Elvira, 2005). Para una completa revisión de las técnicas de medición, su desarrollo y sus limitaciones, el lector es referido al trabajo de Sommerville (2007).

La técnica NIRS, por otro lado, parece interesante en la evaluación de lanas por los grandes volúmenes que se producen y transan a escala mundial, por la necesidad de evaluar lanas sucias, no procesadas y ante la posibilidad de apoyar en forma efectiva programas de mejoramiento ovino (Hammersley y Townseed, 2008). Este método se basa en que los enlaces del hidrógeno con otros átomos constituyentes de la materia orgánica (C, O, N, etc.) absorben energía en sectores específicos, en el rango NIR del espectro electromagnético, por lo que la absorbancia a cualquier longitud de onda es proporcional al número o concentración de moléculas absorbentes presentes en el camino recorrido por la radiación (Osborne et al., 1993; Cozzolino, 2002). Entre otras ventajas, esta técnica permite hacer determinaciones rápidas y múltiples, de bajo costo, mínimo pre-tratamiento de las muestras y sin utilizar reactivos químicos (Murray, 1993). Por otra parte, las limitaciones son los altos costos de inversión en equipos, capacitación y la necesidad de desarrollar calibraciones a través de métodos de análisis tradicionales que deben ser confiables y estar disponibles (Alomar y Fuchslocher, 1998). Existen antecedentes previos, principalmente en Australia, del uso de NIRS para evaluar el diámetro y otras variables en lanas pero en mayor medida orientados a lanas limpias (Hammersley y Townseed, 2008). Los resultados con lanas sucias no siempre han sido alentadores (Cozzolino et al., 2005).

El objetivo de este trabajo fue evaluar la técnica NIRS para predecir variables de interés económico en lana, utilizando muestras provenientes de ovejas Corriedale y Merino Multipropósito de la Región de Magallanes, Chile.

\section{MATERIAL Y MÉTODOS}

Se utilizaron muestras de lana sucia provenientes de ovejas adultas de raza Merino Multipropósito y Corriedale (22 muestras de cada raza), provenientes de la Estancia Cerro Negro (km 187 de la ruta nacional número 9), Región de Magallanes y Antártica Chilena. Las muestras se obtuvieron del sector costal medio, que representa el promedio del vellón. Para cada muestra se crearon sub-muestras para los diferentes análisis: de referencia y NIRS.

El análisis de referencia se realizó por dos métodos secundarios en laboratorios de Punta Arenas y de Nueva Zelandia. En Punta Arenas la lana se evaluó en el laboratorio del Consorcio Ovino S.A. a través de un equipo OFDA 2000 (BSC Electronics PTY Ltd.) que se basa en el análisis de imágenes digitalizadas. En Nueva Zelandia, las muestras fueron evaluadas en el laboratorio certificado de New Zeland Wool Testing Authority (NZWTA), mediante el método Sirolan Laserscan (AWTA Ltd.), técnica basada en el análisis de la intercepción de luz de fibras individuales suspendidas en una mezcla de alcohol y agua, mediante tecnología láser.

Las variables evaluadas mediante los métodos de referencia y posteriormente predichas por NIRS para cada muestra fueron: diámetro medio de la fibra, rendimiento al lavado, resistencia, factor de confort y largo de mecha.

A las sub-muestras destinadas al análisis NIRS se les colectó el espectro en triplicado, para luego almacenar el espectro promedio. Cada sub-muestra se insertó sin preparación previa, en cápsulas circulares metálicas, de $35 \mathrm{~mm}$ de diámetro y $10 \mathrm{~mm}$ de profundidad con ventana de cuarzo. La lana se ordenó de modo que quedaran ambos extremos de las fibras visibles. La lectura espectral se realizó en el rango visible e infrarrojo cercano (400-2500 nm, cada $2 \mathrm{~nm}$ ) con un monocromador NIRSystems 6500. Las cápsulas se pusieron en un módulo de rotación, que permite que el espectro sea colectado en un área más amplia de la muestra, tomándose las lecturas mientras ésta gira. Para cada espectro, se tomaron 32 lecturas, además de otras tantas a una placa de cerámica blanca que actúa como referencia para el equipo. 
Para la colección de espectros, manejo de los archivos y desarrollo posterior de las ecuaciones de calibración, se utilizó el software WINISI II (Infrasoft International). Las calibraciones consistieron en ajustar modelos de regresión múltiple entre los datos espectrales y los de referencia. Previamente, los espectros se sometieron a varios tratamientos matemáticos, consistentes en un primer o segundo orden de diferenciación (pseudo derivada) que es una sustracción de los datos espectrales a intervalos definidos, junto a una suavización (mediante promedio) de diferentes segmentos (cuatro, ocho o doce nm) espectrales. Además, se evaluó la aplicación (o no) de un tratamiento de corrección de dispersión de luz (scatter correction). Para esto los espectros se sometieron al método combinado de la varianza normal estándar (SNV) y el método "Detrend" (SNV \& detrend). El método de regresión utilizado fue el de cuadrados mínimos parciales modificados (MPLS), método multivariados que estandariza cada factor calculado, dividiéndolo por el valor residual promedio, previo al cálculo del próximo factor.

Dado el bajo número de muestras disponibles, las ecuaciones desarrolladas se sometieron a una validación cruzada, donde el set de muestras fue dividido en cinco grupos, dejando uno fuera para ser predicho mediante la ecuación desarrollada con los cuatro restantes. Este proceso fue repetido para cada uno de los grupos, calculándose en cada ciclo un parámetro de certidumbre e incertidumbre, obteniéndose finalmente un coeficiente de determinación para la validación cruzada $\left(\mathrm{R}^{2} \mathrm{CV}\right)$ y un error estándar de validación cruzada (SECV). Además, el proceso incluyó dos pases de eliminación de muestras aberrantes ("outliers") del tipo " $\mathrm{T}$ ", es decir, que presenten residuales anormalmente elevados entre los valores de referencia y las predicciones NIRS del modelo de regresión desarrollado. El valor crítico para outliers T, se fijó en 2,5.

Como criterio principal para seleccionar ecuaciones se utilizó el SECV y la relación RPD, que es el resultado de dividir la desviación estándar de los datos de referencia por el SECV, considerándose adecuado un RPD igual o mayor a tres (Williams, 2001).

\section{RESULTADOS Y DISCUSIÓN}

Los valores de referencia obtenidos para las variables estudiadas para cada raza (22 Corriedale y 22 MPM) se muestran en al Cuadro 1.

Del laboratorio de Nueva Zelandia se obtuvo el diámetro medio de la fibra (DMF), rendimiento al lavado,

Cuadro 1. Datos de referencia separados por raza para las variables lanimétricas obtenidos en los laboratorios del Consorcio Ovino (CO) y del New Zealand Wool Testing Authority (NZ).

Table 1. Reference data separated by breed for the wool quality variables obtained in the laboratories of Consorcio Ovino (CO) and New Zealand Wool Testing Authority (NZ).

\begin{tabular}{|c|c|c|c|c|c|c|}
\hline & $\mathrm{N}^{\circ}$ muestras & Mínimo & Máximo & Media & $\mathrm{DE}$ & $\mathrm{CV}(\%)$ \\
\hline \multicolumn{7}{|l|}{ Corriedale } \\
\hline DMFNZ $(\mu \mathrm{m})$ & 22 & 26,7 & 34,6 & 30,97 & 2,08 & 6,72 \\
\hline DMFCO $(\mu \mathrm{m})$ & 22 & 26,6 & 34,1 & 30,07 & 1,89 & 6,29 \\
\hline RLNZ (\%) & 22 & 65,5 & 86,9 & 79,41 & 4,90 & 6,17 \\
\hline Resistencia & 22 & 20 & 62 & 44,36 & 10,59 & 23,87 \\
\hline $\mathrm{FC}$ & 22 & 25,8 & 67,65 & 44,82 & 10,78 & 24,05 \\
\hline LM & 22 & 76 & 134 & 98,64 & 12,28 & 12,45 \\
\hline \multicolumn{7}{|l|}{ MPM } \\
\hline DMF NZ $(\mu \mathrm{m})$ & 22 & 17,1 & 24,3 & 20,95 & 1,86 & 8,88 \\
\hline DMF CO $(\mu \mathrm{m})$ & 22 & 17 & 22,7 & 20,60 & 1,49 & 7,23 \\
\hline RL NZ (\%) & 22 & 73,6 & 88,3 & 82,70 & 3,71 & 4,49 \\
\hline Resistencia NZ (Newton/kilotex) & 22 & 14 & 65 & 38,50 & 11,82 & 30,70 \\
\hline FC NZ $(\%<30 \mu \mathrm{m})$ & 22 & 85,2 & 99,9 & 96,49 & 4,12 & 4,27 \\
\hline Largo mecha NZ (nm) & 22 & 63 & 121 & 98,50 & 14,95 & 15,18 \\
\hline
\end{tabular}

DMF: diámetro medio de fibras; RL: rendimiento al lavado; Resistencia (Newton/kilotex); FC: factor de confort (\% de fibras con diámetro < $30 \mu \mathrm{m}$ ); LM: Largo de mecha (mm); DE: desviación estándar; CV (\%): coeficiente de variación (DE/Media). 
resistencia a la tracción, factor de confort (FC) y largo de mecha. Por su parte, el laboratorio del Consorcio Ovino entregó el valor de DMF. Es importante señalar que ambos métodos de análisis, Sirolan Laserscan y OFDA 2000 obtuvieron cifras similares para el DMF, aunque los resultados de NZWTA son levemente superiores y con una leve mayor variabilidad. El conjunto de muestras MPM presenta un menor DMF, siendo mejor (más finas) que la lana Corriedale, con aproximadamente 10 micras menos. Las muestras de lana Corriedale se aproximan, con máximos algo superiores, a los valores de diámetro establecidos por la Asociación Argentina de Criadores Corriedale, que oscila entre 27 y 32 micras. Las lanas Merino de la Patagonia Argentina presentan promedios cercanos a 19 (Mueller et al., 2001). Los MPM del presente trabajo poseen un micronaje promedio de 19 a 21 micras, lo que las hace comparables a las Merino anteriores, aunque levemente más gruesas.

En cuanto al rendimiento al lavado, MPM obtiene un valor mayor en 3,3 unidades de porcentaje, en relación a Corriedale. Por su parte, la lana Corriedale se muestra superior en la resistencia a la tracción, resistiendo en promedio 5,86 (newton/kilotex) más que MPM, lo que es coherente con su mayor diámetro. Finalmente, en el factor de confort, entendido como el porcentaje de lanas con diámetro medio inferior a $30 \mu \mathrm{m}$, se observa una gran diferencia a favor del MPM, lo cual es esperable debido a su menor diámetro de fibras y a que incluso en las muestras que se encuentran en el extremo superior del rango, el diámetro promedio es inferior a los $30 \mu \mathrm{m}$.

En el desarrollo de modelos de predicción es deseable tener una amplia dispersión de los valores de las variables a predecir, que represente la diversidad que se pueda encontrar para el material en estudio y que facilite un mejor ajuste en el análisis de regresión (Mu- rray, 1988). En este trabajo, la variabilidad, representada por el coeficiente de variación (CV), es limitada si se consideran los valores por separado para cada raza, con $\mathrm{CV}$ inferiores a $10 \%$ para diámetro (ambos laboratorios) y rendimiento al lavado. Esto es esperable y responde a los esfuerzos de selección dentro de cada raza, principalmente en lo referente al diámetro. También se observa un bajo $\mathrm{CV}$ para el factor de confort en MPM, pero no en Corriedale. Sin embargo, si se consideran las razas en su conjunto, que fue la forma en que se desarrollaron las calibraciones, la dispersión de los datos es bastante más amplia para todas las variables, a excepción del rendimiento al lavado, que presentó un coeficiente de variación inferior a 10\%.

\section{Calibraciones NIRS}

La hipótesis central de este trabajo indica que la tecnología NIRS tiene la capacidad de predecir variables relevantes de calidad de lana, en especial el diámetro de las fibras, por su importancia económica. En el Cuadro 2 se caracterizan de acuerdo a sus parámetros estadísticos las ecuaciones seleccionadas para aquellas variables lanimétricas (diámetro, factor de confort y rendimiento al lavado) que podrían ser predichas en forma confiable con esta tecnología. Todas presentaron coeficientes de determinación de al menos 0,9 (RL) o muy cercanos a la unidad (DMF en ambos laboratorios). Los indicadores estadísticos de certidumbre $\left(\mathrm{R}^{2}\right)$ e incertidumbre (SEC) de la etapa de calibración sufren un deterioro (sus valores disminuyen y aumentan, respectivamente) al pasar a la etapa de validación cruzada ( $\mathrm{R}^{2} \mathrm{CV}$ y $\left.\mathrm{SECV}\right)$. Esto es esperable, ya que en la etapa de calibración, las mismas muestras que participan en el modelo de regresión son predichas por éste.

Cuadro 2. Indicadores estadísticos de las calibraciones seleccionadas.

Table 2. Statistic indicators of the selected calibrations.

\begin{tabular}{lccccccc}
\hline \multicolumn{1}{c}{ Variable } & $\mathrm{R}^{2}$ & $\mathrm{SEC}$ & $\mathrm{R}^{2} \mathrm{CV}$ & $\mathrm{SECV}$ & $\mathrm{PROM}$ & $\mathrm{DE}$ & $\mathrm{RPD}$ \\
\hline DMF (CO) & 0,99 & $0,40 \mu \mathrm{m}$ & 0,94 & $1,24 \mu \mathrm{m}$ & $24,84 \mu \mathrm{m}$ & $4,90 \mu \mathrm{m}$ & 3,96 \\
DMF (NZ) & 0,97 & $0,95 \mu \mathrm{m}$ & 0,90 & $1,65 \mu \mathrm{m}$ & $25,66 \mu \mathrm{m}$ & $5,35 \mu \mathrm{m}$ & 3,24 \\
FC & 0,93 & $7,36 \%$ & 0,91 & $8,26 \%$ & $71,31 \%$ & $27,03 \%$ & 3,27 \\
RL & 0,90 & $1,44 \%$ & 0,89 & $1,54 \%$ & $81,29 \%$ & $4,55 \%$ & 2,95 \\
\hline
\end{tabular}

$\mathrm{R}^{2}$ : Coef. de determinación de la calibración

SEC: Error estándar de calibración

$\mathrm{R}^{2} \mathrm{CV}$ : Coef. de determinación de validación cruzada

SECV: Error estándar de validación cruzada

PROM: Promedio de los datos de referencia de las muestras;

DE: Desviación estándar de los datos de referencia;

RPD: relación DE/SECV

DMF (CO): Diámetro promedio de fibras (lab. Consorcio Ovino); DMF (NZ): Diámetro promedio de fibras (lab. N. Zelandia); RL: Rendimiento

al lavado; FC: Factor de confort. 
En cambio, la validación cruzada equivale a un tipo de validación externa, pero que evita tener que reducir el número de muestras que participan en la calibración, si se considera el proceso completo. Esta pérdida de ajuste puede ser proporcionalmente diferente, dependiendo del parámetro y de la variable en cuestión. Así, las calibraciones que sufren menos deterioro entre ambas etapas son aquellas para FC y RL.

Tres de las cuatro calibraciones presentan relaciones RPD superiores a tres y una (RL) muy cercana $(2,95)$. Concordantemente, todas las ecuaciones seleccionadas presentan un elevado ajuste de los datos predichos con los de referencia, con un $\mathrm{R}^{2} \mathrm{CV}$ muy cercano o superior a 0,9 . Si bien el RL no alcanza el umbral de RPD de 3 para la validación cruzada, el error que presenta en esta etapa (SECV) corresponde a una muy pequeña proporción del promedio de los datos de referencia $(0,02)$, lo cual permitiría una seguridad aceptable desde el punto de vista predictivo. Para el resto de las variables (largo de mecha y resistencia al quiebre) no se obtuvieron resultados alentadores, ya que los $\mathrm{R}^{2}$ de calibración y validación cruzada fueron cercanos a cero y los correspondientes errores de calibración y validación cruzada fueron excesivamente elevados (similares o superiores a la desviación estándar de los datos de referencia).

Todos los modelos de regresión seleccionados se obtuvieron con un segundo orden de sustracción del espectro y solo el RL incluyó corrección de dispersión de luz (SNV y Detrend). A su vez, los tratamientos de los espectros difirieron en la magnitud del intervalo de sustracción y segmento de suavización, que se ubicaron entre 4 y 8 puntos de datos.

En el trabajo realizado por Cozzolino et al. (2005) orientado a predecir mediante NIRS el diámetro de fibras de lana, se obtuvieron calibraciones aceptables para muestras de lana limpia (RPD $>3$ ) pero no para lana sucia (RPD $<1)$. Esto fue así, a pesar de contar con alrededor de 400 muestras de lana sucia y solo 50 de lana limpia. En el presente trabajo, el número de muestras puede ser un factor limitante, siendo deseable incrementar la base de datos en futuros estudios, para cubrir un rango que se aproxime mejor a lo que se puede encontrar en la realidad.

Para ilustrar el grado de ajuste en las predicciones obtenidas, en la Figura 1 se aprecia la distribución de los datos de referencia para el DMF (Consorcio Ovino) y aquellos predichos por NIRS (eje Y) en la mejor calibración obtenida. Se observa que los valores se sitúan muy cercanos a la línea diagonal (igual respuesta, $\mathrm{Y}=\mathrm{X}$ ) con una baja dispersión alrededor de ésta, lo que representa los errores del modelo. Estos errores incluyen aquellos atribuibles a aspectos espectrales, pero también aquellos propios del método de referencia, los que lamentablemente no fueron determinados en este trabajo. Se observa que las muestras tienden a agruparse de acuerdo a



Figura 1. Relación entre los datos de referencia y predichos por NIRS, para DMF (CO) de muestras de lana MPM (rombos) y Corriedale (círculos).

Figure 1. Relationship between reference and NIRS predicted data, for DMF (CO) of MPM (diamonds) and Corriedale (circles) wool samples.

las características raciales de la lana. Así, las lanas MPM (rombos) se ubican en los valores menores de diámetro y las lanas Corriedale (círculos) en los valores mayores.

Este trabajo debe considerarse de carácter exploratorio, ya que se contó con una cantidad limitada de muestras. Una proyección de esta línea de trabajo, debería considerar la incorporación de una mayor cantidad de muestras de lana y, en lo posible, contar con una estimación del error estándar de laboratorio (SEL). Esto permitiría desarrollar una predicción externa y evaluar el modelo obtenido de acuerdo a límites de control definidos. Complementariamente, sería deseable que estas muestras se distribuyan en los sectores menos representados de la escala de diámetro cubierta (23 a $27 \mu \mathrm{m}$ ). Esto se podría lograr incorporando lanas provenientes de cruzas entre MPM y Corriedale, presentes en la zona austral, o de otras razas apropiadas.

\section{CONCLUSIONES}

De acuerdo a los resultados obtenidos, se concluye que la tecnología NIRS tiene la capacidad de predecir algunas variables relevantes de calidad de lana, tales como el diámetro promedio de fibras, el factor de confort y el rendimiento al lavado. De este modo, en forma rápida, sin ninguna preparación previa de la muestra y con un grado aceptable de error, es posible obtener una predicción múltiple de algunos atributos de la lana que revisten gran importancia económica. 


\section{REFERENCIAS}

Alomar, D., Fuchslocher, R., 1998. Fundamentos de la espectroscopía de reflectancia en el infrarrojo cercano (NIRS) como método de análisis de forrajes. Agro Sur 26 (1), 88104. http://mingaonline.uach.cl/scielo.php?pid=S030488021998000100011\&script=sci_arttext.

Asociación Argentina Criadores de Corriedale., 2007. Raza Corriedale. Sitio Argentino de Producción Animal. <http://www.produccion-animal.com.ar/produccion_ ovina/razas_ovinas/74-raza_corriedale.pdf> (acceso, 09.02.2015).

Buxade, C., 1996. Zootecnia. Bases de Producción Animal. Tomo VIII Producción Ovina. Ediciones Mundi-Prensa, Madrid.

Cozzolino, D., 2002. Uso de la espectroscopía de reflectancia en el infrarrojo cercano (NIRS) en el análisis de alimentos para animales. Agrociencia 6(2), 25-32. http:// www.fagro.edu.uy/ agrociencia/index.php/directorio/article/viewFile/543/452.

Cozzolino, D., Montossi, F., San Julian, R., 2005. The use of visible (VIS) and near infrared (NIR) reflectance spectroscopy to predict fibre diameter in both clean and greasy wool samples. Animal Science 80, 333-337.

Elvira, M., 2005. Características de lana Merino e importancia en el procesamiento industrial. Asociación Argentina de criadores de Merino (Argentina) Boletín Año XIII № 49, pp. 231-238.

Fundación Chile., 2002. Tópicos de Producción Ovina en el secano central. Gobierno de Chile, Ministerio de Agricultura. http://es.scribd.com/doc/87856454/CorderoSecanoCentral (acceso, 15.06.2012).

Hammersley, M.J., Townsend, P.E., 2008. NIR Analysis of Wool, in: Burns, D., Ciurczak, E. (Eds.), Handbook of near-infrared analysis. CRC Press, Boca Raton, pp. 465-478.

International Wool Textile Organisation (IWTO)., 2012. Wool Roadmap. http://www.iwto.org/uploaded/projects/ro- admap/iwto_rm_spanish.pdf (acceso, 15.06.2012).

Itenge, T.O., Hickford, J.G.H., Forrest, J.G.H., McKenzie, G.W., Frampton, C.M., 2009. Improving the quality of wool through the use of gene markers. South African Journal of Animal Science 39(1), 219-223. <http://dx.doi. org/10.4314/sajas.v39i1.61191>.

Latorraca, A., 2005. Esquila desmaneada secuencial. Memorias del VII curso de Actualización en Producción Ovina, EEA Bariloche, INTA, pp. 133-144.

Mueller, J., Duga, L., Giraudo, C., Bidinost, F., 2001. Calidad de vellones en una majada Merino de la Patagonia. Revista de Investigaciones Agropecuarias 30, 101-113.

Murray, I., 1988. Aspects of the interpretation of near infrared spectra. Food Science and Technology Today 2, 135-139.

Murray, I., 1993. Forage analysis by near infrared spectroscopy, in: Davies, A., Baker, R.D., Grant, S.A., Laidlaw, A.S. (Eds.), Sward herbage measurement handbook. British Grassland Society, Maidenhead, pp. 285-312.

Osborne, B.G., Fearn, T., Hindle, P.H., 1993. Near infrared spectroscopy in food analysis. $2^{\text {nd }} \mathrm{ed}$. Longman Scientific and Technical. Harlow.

Sacchero, D., 2005. Utilización de medidas objetivas para determinar calidad en lanas. Sitio Argentino de Producción Animal. <http://www.produccionanimal.com.ar/ produccion_ovina/produccion_ovina_lana/69-calidad_ lanas.pdf> (acceso, 09.02.2015).

Sommerville, P., 2007. Fundamental principles of fibre finness measurement. Australian Wool Testing Authority Ltd. Guildford.

The Livestock Conservancy., 2015. <http://www.livestockconservancy.org/index.php/resources/internal/heritage-sheep> (acceso, 03.03.2015)

Williams, P.C., 2001. Implementation of near infrared technology, In: Williams, P.C., Norris, K.H. (Eds.), Near infrared technology in the agricultural and food industries. American Association of Cereal Chemists. St Paul, Minnesota, pp. 145-171. 